\title{
Palliative pelvic radiotherapy of symptomatic incurable rectal cancer - a systematic review
}

\author{
MARTE GRØNLIE CAMERON ${ }^{1}$, CHRISTIAN KERSTEN ${ }^{1}$, INGVILD VISTAD ${ }^{2}$, \\ SOPHIE FOSS $\AA^{3} \&$ MARIANNE GRØNLIE GUREN ${ }^{3}$
}

${ }^{1}$ Center for Cancer Treatment, Sørlandet Hospital Trust, Kristiansand, Norway, ${ }^{2}$ Department of Obstetrics and Gynecology, Sorlandet Hospital Trust, Kristiansand, Norway and ${ }^{3}$ Department of Oncology, Oslo University Hospital, Norway

\begin{abstract}
Background. Locally advanced and recurrent rectal cancers frequently cause pelvic morbidity including pain, bleeding and mass effect. Palliative pelvic radiotherapy is used to relieve these symptoms and delay local progression. There is no established optimal radiotherapy regimen and clinical practices vary. Our aim was to review the efficacy and toxicity of palliative pelvic radiotherapy of symptomatic rectal cancer and to evaluate different fractionation schedules, based on published literature. Material and methods. Systematic literature searches of Medline, Embase and Cochrane databases were performed through 2011. Studies reporting symptomatic response or quality of life (QOL) after palliative radiotherapy for rectal or rectosigmoid cancer were eligible. Results. Twenty-seven studies were included, of which 23 were retrospective reviews. There were no patient-reported outcomes or QOL assessments. There were large variations in applied radiotherapy regimens. Pooled overall symptom response rate was $75 \%$ and positive responses were reported for pain ( $78 \%$ ), bleeding and discharge $(81 \%)$, mass effect $(71 \%)$ and other pelvic symptoms $(72 \%)$. Toxicity results were not evaluable. Conclusion. Palliative pelvic radiotherapy for symptomatic rectal cancer appears to provide relief of a variety of pelvic symptoms, although there is no documented optimal radiotherapy regimen in this context. There is inadequate evidence regarding onset, duration and degree of symptom palliation, QOL and associated toxicity with this treatment and prospective studies are therefore needed.
\end{abstract}

Locally advanced primary and recurrent rectal and rectosigmoid cancers have the potential to produce significant pelvic morbidity including pain, obstruction, tenesmus, hemorrhage and discharge. Systemic oncologic treatments, which have prolonged the median survival of patients with advanced colorectal cancer by up to two years [1], usually have a positive effect on the primary tumor [2]. However, a subgroup of patients still experiences the burden of a growing pelvic mass unsuited for surgical excision. Prolonged survival, relief of symptoms and sustained quality of life (QOL) are of great importance for these patients and palliative external beam radiotherapy (EBRT) is often used for these purposes.

Population-based studies report a general underutilization of palliative radiotherapy [3]. Among the proposed explanations are a lack of evidence to support its use and concern regarding toxicity [4]. Among patients with advanced and incurable cancers in need of palliative radiotherapy, there is a trend toward using short-course, hypofractionated regimens that have been proven efficacious while significantly reducing time spent in treatment $[5,6]$.

There is currently no consensus on how palliative pelvic EBRT of rectal cancer should optimally be delivered. Such a standard should be based on documentation of patient-reported QOL and symptom relief weighed against the burden of the treatment. Among frail and elderly patients with primarily inoperable rectal cancer, hypofractionated preoperative radiotherapy has been shown to downsize the tumor, with acceptable toxicity [7-9].

Correspondence: M. Grønlie Cameron, Sørlandet Sykehus HF, Serviceboks 416, 4604 Kristiansand, Norway. Tel: +47 38146600 . Fax: + 4738146601. E-mail: marte.cameron@sshf.no 
In 1996 Wong et al. authored a review of the role of radiotherapy in the management of pelvic recurrence of rectal cancer [10] including both preoperative and palliative radiotherapy, and subgroups treated with chemoradiotherapy. Their review focused specifically on recurrent rectal cancers, which are becoming less frequent in the era of total mesorectal excision (TME) [11]. Their conclusion was that pelvic radiotherapy had value in relieving symptoms, but that the optimal dose and fractionation for palliative treatment of recurrent rectal cancer could not be determined.

The aim of the present systematic review was to evaluate published studies describing the effects of palliative EBRT of symptomatic, incurable primary and recurrent rectal (and rectosigmoid) cancer in order to determine its effect on symptom palliation and QOL. In addition, we aimed to review the reported toxicity in order to clarify the risk-benefit balance and finally, to evaluate published treatment schedules in order to determine whether there exists an optimal dose or fractionation scheme. Implications of these findings for clinical practice and future directions of research are discussed.

\section{Material and methods}

This review is based on a scientific research protocol describing the aims and methods used. Within limitations imposed by the nature of the research in this field, this synthesis is reported according to the Preferred Reporting Items for Systematic Reviews and Meta-Analyses: The PRISMA statement [12].

\section{Search strategy}

Searches of the Medline, Embase and Cochrane library databases were performed through December, 2011. The following example illustrates the search strategy (MESH terms) used in Medline: (radiotherapy $\mathrm{OR}$ radiation OR radiation oncology) AND (palliative care or terminal care) AND colorectal neoplasms. Titles/abstracts were screened by four authors (MC, MG, CK, IV), and full text copies of all studies of potential relevance, including review articles, were obtained. Further studies were identified manually from the reference lists of the articles reviewed in full-text (MC).

\section{Eligibility criteria}

Published studies of palliative pelvic EBRT of rectal and rectosigmoid cancer that reported symptom response or QOL were considered eligible for inclusion. Studies that included the target population as a subgroup were included as long as results of palliative pelvic radiotherapy for the subgroup could be identified. All study designs (other than case reports and reviews) were eligible. Studies with weaker methodology (i.e. non-randomized, retrospective studies) were included in the review in order to ensure as complete an overview of the existing evidence as possible. Studies evaluating radiotherapy combined with other tumor-directed treatment modalities or re-irradiation were excluded. Studies published in European languages were eligible and translations were carried out when necessary.

\section{Evaluation of studies}

The evaluation of potential studies at the full-text level was performed independently by four of the authors (MC, MG, CK, IV) and final selection was based on consensus. Articles were evaluated by each author using a study selection form based on the Cochrane group's criteria [13], which were altered and pilot tested for this specific purpose. There is no universally accepted and validated tool for assessing the "quality" of retrospective and observational studies [14]. Numeric scoring of the quality of the original articles was therefore abandoned and the criteria instead focused qualitatively on the internal validity of the individual studies and included an assessment of the risk of bias both at the study and outcome levels.

\section{Data extraction and management}

Data regarding study characteristics and the outcomes of interest (symptom response, QOL, and toxicity) were extracted independently by two reviewers (MC, MG) and results were compared. The final data set is based on consensus. Meta-analysis was not feasible because of the heterogeneity of study populations, treatments, and outcomes. Data were therefore described in table form, using summary headings. Symptomatic response rates, according to the original authors' own definitions, at variable time points after palliative pelvic radiotherapy were dichotomized (response versus no response), pooled and presented descriptively. An attempt has been made to link the quality of the included studies to the interpretation of their results [12]; retrospective reports being interpreted with caution.

\section{Results}

\section{Study selection}

Results of the study selection process are outlined in Figure 1. A total of 27 studies were included in the review. 


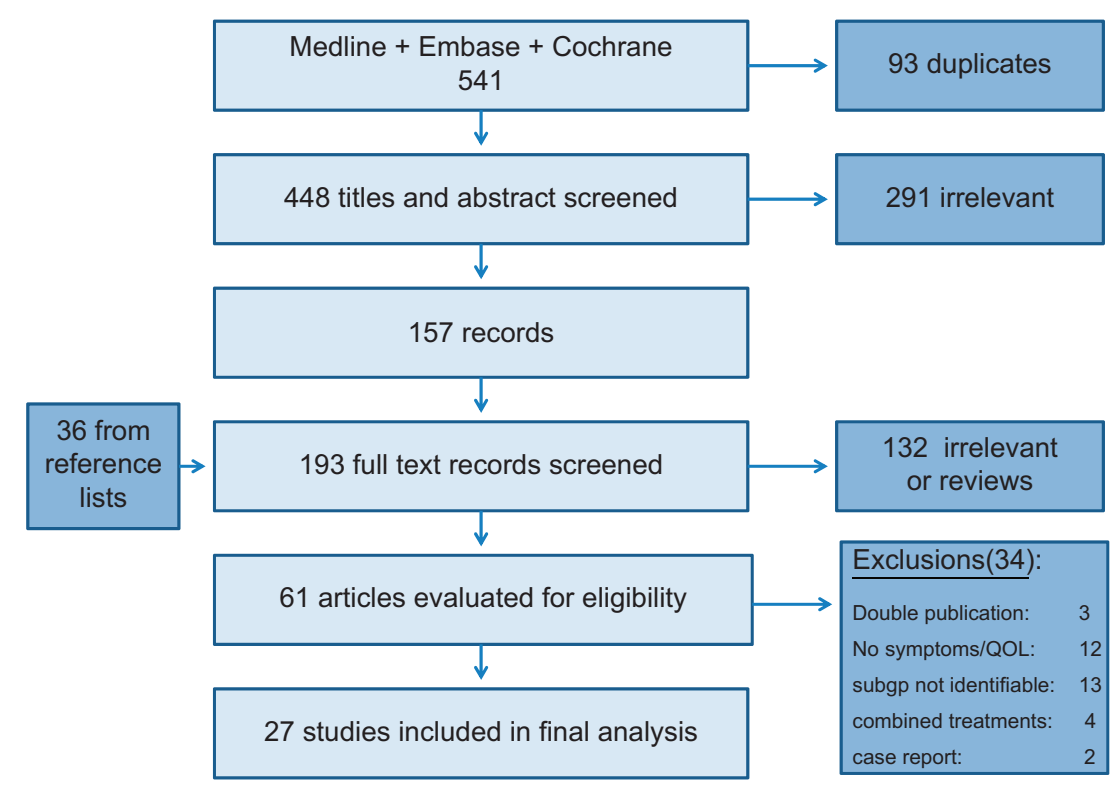

Figure 1. Study flowchart.

\section{Study characteristics}

An overview of the characteristics of the 27 studies included in the final analysis is provided in Table I. Four prospective studies were included. Of these, two were randomized controlled trials in which the population given palliative pelvic radiotherapy served as the control group $[15,16]$, one was nonrandomized [17], and one was an observational study including patients assessed both prospectively and retrospectively, but given similar treatments [18]. The remaining 23 studies were retrospective chart reviews. In those retrospective studies where data collection methods were reported, symptom data had been extracted from physicians' clinical notes. One study addressed quality (validity, reliability) of the retrospective data extraction procedure [19]. The total number of relevant patients included in this review is 1759 . There was a mean of 65 (range 9-189) relevant patients per study. Among the 18 studies that reported the time span of the treatments being evaluated, there was a median duration of eight years (range 2-27), the oldest referring to patients treated in the 1930s [20] and the newest reporting treatments given up to 1991 [16].

\section{Patient characteristics and symptoms}

Three studies included only patients with primary rectal or rectosigmoid tumors [18,20,21], 14 included only patients with recurrent or residual pelvic disease [22-35] and the remaining 10 studies included a combination of the two. In 15 of the 27 studies, the population of interest was a subgroup of a larger study. The majority of studies included patients with distant metastases, although not all patients had been investigated to this end [27]. The most commonly reported symptoms were pain, bleeding, mass and "other rectal disorders" including among others, discharge and perineal nodules. Two studies did not specify which symptoms were evaluated, but reported an overall palliative effect.

\section{Radiotherapy dose and fractionation}

Radiotherapy method, dose, schedule, and target definitions varied between, and in many cases also within, studies (Table I; Figure 2). Treatments were given both as single fractions and more commonly, fractionated over several weeks (up to nine weeks). The most commonly used fraction size was $2 \mathrm{~Gy}$ (range 1.5-10 Gy) and total doses ranged from 5 to $70 \mathrm{~Gy}$, most often in the range of $30-60 \mathrm{~Gy}$. Two studies $[15,18]$ gave the same total dose to all patients in the relevant subgroup, while the largest range of total doses within a single study was $15-70$ Gy [24]. Some authors did not clearly indicate the distribution of radiotherapy doses given $[27,32,36,37]$. Biologically effective doses (BED) could not be calculated for comparative purposes given limited data regarding radiotherapy delivery in many studies. Variability and distribution of total radiotherapy doses have remained stable over the more than 50 year period covered by the publications.

\section{Symptom response}

The proportion of positive symptom responses according to the authors' own definitions are shown in Tables II and III. Overall symptomatic response 
Table I. Characteristics of studies of palliative pelvic radiotherapy of rectal cancer.

\begin{tabular}{|c|c|c|c|c|c|}
\hline Author/year & $\begin{array}{l}\text { Study design and } \\
\text { treatment period }\end{array}$ & Participants & $\begin{array}{l}\text { Radiotherapy (Dose range/ } \\
\text { fraction size/treatment } \\
\text { period) }\end{array}$ & $\begin{array}{l}\text { Relevant } \\
\text { outcome }\end{array}$ & Follow-up \\
\hline $\begin{array}{l}\text { Allum [18] } \\
1987\end{array}$ & $\begin{array}{l}\text { Retrospective } \\
1983-1985\end{array}$ & $\begin{array}{l}\mathbf{N}=18 \text { inoperable recurrent } \\
\text { CRC }\end{array}$ & $\begin{array}{l}\text { 30-45 Gy/3 Gy } \\
5-10 \text { Gy/single fraction } \\
\text { (some repeated) }\end{array}$ & $\begin{array}{l}\text { Pain relief, } \\
\text { 3-point scale }\end{array}$ & NR \\
\hline $\begin{array}{l}\text { Carlsson [14] } \\
1986\end{array}$ & $\begin{array}{l}\text { Pro, non- } \\
\text { randomized, } \\
\text { controlled trial, } \\
\text { NR }\end{array}$ & $\begin{array}{l}79 \text { inoperable or recurrent rectal } \\
\text { cancer } \\
\text { RSN }=47 \text { given EBRT }\end{array}$ & 30 or $45 \mathrm{~Gy} / 2 \mathrm{~Gy}$ & $\begin{array}{l}\text { Pain relief, } \\
\text { 4-point scale }\end{array}$ & $1 \mathrm{mo}$ \\
\hline $\begin{array}{l}\text { Ciatto [19] } \\
1982\end{array}$ & $\begin{array}{l}\text { Retrospective } \\
1956-1976\end{array}$ & $\begin{array}{l}\mathbf{N}=108 \text { recurrent rectal } \\
\text { or } \mathrm{RS} \text { cancer } \\
\mathbf{R S N}=\mathbf{9 0} \text { symptomatic }\end{array}$ & $\begin{array}{l}35-50 \mathrm{~Gy} / 2 \mathrm{~Gy} / 4-5 \mathrm{wk} \\
\text { Boost of } 10-15 \mathrm{~Gy} \text { in a few } \\
\text { cases }\end{array}$ & $\begin{array}{l}\text { Complete } \\
\text { regression of } \\
\text { symptoms }\end{array}$ & $\begin{array}{l}\text { Minimum } 5 \text { yr } \\
\text { None lost }\end{array}$ \\
\hline $\begin{array}{l}\text { DeRenzis [20] } \\
1986\end{array}$ & $\begin{array}{l}\text { Retrospective } \\
1981-1984\end{array}$ & $\begin{array}{l}\mathbf{N}=35 \text { inoperable or recurrent } \\
\text { rectal cancer }\end{array}$ & $\begin{array}{l}\text { Palliation: } 40-50 \text { Gy } \\
\text { Cure: }>50 \text { Gy }\end{array}$ & $\begin{array}{l}\text { Symptom relief, } \\
\text { not defined }\end{array}$ & $\begin{array}{l}35 / 35-2 \mathrm{yr} \\
21 / 35-3 \mathrm{yr}\end{array}$ \\
\hline $\begin{array}{l}\text { Dobrowsky [21] } \\
1985\end{array}$ & $\begin{array}{l}\text { Retrospective } \\
1975-1982\end{array}$ & $\begin{array}{l}58 \text { rectal cancer recurrence } \\
\mathbf{R S N}=\mathbf{3 8} \text { symptomatic }\end{array}$ & $\begin{array}{l}\text { 15-70 Gy (some given split } \\
\text { course) }\end{array}$ & $\begin{array}{l}\text { Pain relief, } \\
\text { 4-point scale }\end{array}$ & $\begin{array}{l}\text { Minimum } 2 \text { yr } \\
\text { None lost }\end{array}$ \\
\hline $\begin{array}{l}\text { Gescher [22] } \\
1987\end{array}$ & $\begin{array}{l}\text { Retrospective } \\
1977-1983\end{array}$ & $\begin{array}{l}\mathbf{N}=\mathbf{6 1} \text { inoperable recurrent } \\
\text { rectal cancer }\end{array}$ & $50-70 \mathrm{~Gy} / 2-2.5 \mathrm{~Gy} / 5-9 \mathrm{wk}$ & $\begin{array}{l}\text { Symptom relief, } \\
\text { dichotomized } \\
\text { and PI }\end{array}$ & NR \\
\hline $\begin{array}{l}\text { Guiney [23] } \\
1999\end{array}$ & $\begin{array}{l}\text { Retrospective } \\
1981-1990\end{array}$ & $\begin{array}{l}57 \text { residual rectal or } \mathrm{RS} \text { cancer } \\
\mathbf{R S N}=\mathbf{1 7} \text { palliative treatment, } \\
\text { symptomatic }\end{array}$ & $\begin{array}{l}45 \mathrm{~Gy} / 3 \mathrm{~Gy} / 4 \mathrm{wk} \\
30 \mathrm{~Gy} / 5 \mathrm{~Gy} / 4 \mathrm{wk}\end{array}$ & $\begin{array}{l}\text { Symptom relief, } \\
\text { 3-point scale }\end{array}$ & $\begin{array}{r}\text { Median } 49 \text { mo } \\
\text { (range 5-80) }\end{array}$ \\
\hline $\begin{array}{l}\text { James [24] } \\
1983\end{array}$ & $\begin{array}{l}\text { Retrospective } \\
\text { Period NR }\end{array}$ & $\begin{array}{l}143 \text { symptomatic recurrent } \\
\text { rectal cancer } \\
\mathbf{R S N}=\mathbf{1 1 9} \text { evaluable }\end{array}$ & $<10 \mathrm{~Gy}$ to $>20 \mathrm{~Gy}$ & $\begin{array}{l}\text { Symptom relief, } \\
\text { 3-point scale }\end{array}$ & $\begin{array}{l}\text { NR. } 24 \text { lost to } \\
\text { follow-up }\end{array}$ \\
\hline $\begin{array}{l}\text { Kimmig [25] } \\
1989\end{array}$ & $\begin{array}{l}\text { Retrospective } \\
1979-1985\end{array}$ & $\mathbf{N}=\mathbf{7 4}$ recurrent $\mathrm{CRC}$ & $\begin{array}{l}60-66 \text { Gy } \\
\text { Perineal affection received } \\
\quad \text { additional } \mathrm{MeV}\end{array}$ & $\begin{array}{l}\text { Pain relief, } \\
\text { dichotomized }\end{array}$ & $2-8 \mathrm{yr}$ \\
\hline $\begin{array}{l}\text { Murdock [26] } \\
1964\end{array}$ & $\begin{array}{l}\text { Retrospective } \\
1957-1962\end{array}$ & $\begin{array}{l}\mathrm{N}=13 \text { perineal recurrence of } \\
\mathrm{CRC} \\
\mathbf{R S N}=\mathbf{9} \text { radiotherapy alone }\end{array}$ & $24-56 \mathrm{~Gy} / 2 \mathrm{~Gy} / 1-4 \mathrm{wk}$ & $\begin{array}{l}\text { Symptom relief, } \\
\text { 4-point scale }\end{array}$ & NR \\
\hline $\begin{array}{l}\text { Murphy [27] } \\
1964\end{array}$ & $\begin{array}{l}\text { Retrospective } \\
1942-1961\end{array}$ & $\begin{array}{l}135 \text { irradiated rectal or RS } \\
\text { cancer } \\
\mathbf{R S N}=127 \text { inoperable or } \\
\text { recurrent }\end{array}$ & $20-60 \mathrm{~Gy} / 2-7 \mathrm{wk}$ & $\begin{array}{l}\text { Palliation, not } \\
\text { defined }\end{array}$ & NR \\
\hline $\begin{array}{l}\text { O'Connell [12] } \\
1982\end{array}$ & $\begin{array}{l}\text { RCT } \\
\text { NR }\end{array}$ & $\begin{array}{l}44 \text { inoperable or recurrent rectal } \\
\text { or RS cancer } \\
\text { RSN }=19 \text { randomized to EBRT } \\
\text { alone (control gp) }\end{array}$ & $\begin{array}{l}50 \mathrm{~Gy} / 2 \mathrm{~Gy} / 7 \mathrm{wk} \\
\text { (split-course) }\end{array}$ & $\begin{array}{l}\text { Pain relief, not } \\
\text { defined }\end{array}$ & NR \\
\hline $\begin{array}{l}\text { Pacini }[28] \\
1986\end{array}$ & $\begin{array}{l}\text { Retrospective } \\
1956-1983\end{array}$ & $\begin{array}{l}\mathrm{N}=143 \text { recurrent rectal or } \mathrm{RS} \\
\text { cancer }\end{array}$ & $35-65$ Gy/2-3 Gy/3-7 wk & $\begin{array}{l}\text { Symptom relief, } \\
\text { dichotomized } \\
\text { and PI }\end{array}$ & $\begin{array}{l}\text { NR. None lost } \\
\text { to follow-up }\end{array}$ \\
\hline $\begin{array}{l}\text { Påhlman [15] } \\
1985\end{array}$ & $\begin{array}{l}\text { Pro \& } \\
\quad \text { retrospective } \\
1979-1983\end{array}$ & $\begin{array}{l}39 \text { inoperable rectal or RS } \\
\text { cancer } \\
\mathbf{R S N}=\mathbf{2 7} \text { symptomatic, } \\
\text { radiotherapy alone }\end{array}$ & $46 \mathrm{~Gy} / 2 \mathrm{~Gy} / 4-5 \mathrm{wk}$ & $\begin{array}{l}\text { Symptom relief, } \\
\text { 3-point scale }\end{array}$ & NR \\
\hline $\begin{array}{l}\text { Ruggieri [29] } \\
1989\end{array}$ & $\begin{array}{l}\text { Retrospective } \\
1976-1985\end{array}$ & $\mathbf{N}=\mathbf{6 8}$ recurrent rectal cancer & $\begin{array}{l}30-60 \mathrm{~Gy} / 1.6-2.5 / 3-8 \mathrm{wk} \\
\text { Some received additional } \\
\text { perineal boost }\end{array}$ & $\begin{array}{l}\text { Pain relief, } \\
\text { 4-point scale }\end{array}$ & $3 \mathrm{mo}$ \\
\hline $\begin{array}{l}\text { Sinha [30] } \\
1989\end{array}$ & $\begin{array}{l}\text { Retrospective } \\
1974-1983\end{array}$ & $\begin{array}{l}48 \text { recurrent rectal or RS cancer } \\
\mathbf{R S N}=\mathbf{2 5} \text { given EBRT alone }\end{array}$ & $\begin{array}{l}\text { Mean } 50 \mathrm{~Gy} / 2 \mathrm{~Gy} / 5-5.5 \mathrm{wk} \\
8 / 25 \text { received additional } 2 \\
\mathrm{Gv} \times 5 \text { boost }\end{array}$ & $\begin{array}{l}\text { Symptom relief, } \\
\text { dichotomized }\end{array}$ & NR \\
\hline $\begin{array}{l}\text { Sklaroff }[31] \\
1973\end{array}$ & $\begin{array}{l}\text { Retrospective } \\
\text { 1961-? }\end{array}$ & $\mathbf{N}=\mathbf{1 0}$ inoperable rectal cancer & $40-50 \mathrm{~Gy}$ & $\begin{array}{l}\text { Symptom relief, } \\
\text { not defined }\end{array}$ & $\begin{array}{l}\text { Minimum } 6 \\
\text { mo }\end{array}$ \\
\hline $\begin{array}{l}\text { Smedal [32] } \\
1967\end{array}$ & $\begin{array}{l}\text { Retrospective } \\
\text { NR }\end{array}$ & $\begin{array}{l}\mathbf{N}=\mathbf{5 0} \text { recurrent rectal or RS } \\
\text { cancer (including } 2 \text { cases of } \\
\text { anal cancer) }\end{array}$ & $20-60 \mathrm{~Gy}$ & $\begin{array}{l}\text { Palliation, } \\
\text { 4-point scale }\end{array}$ & NR \\
\hline $\begin{array}{l}\text { Soleimani }[16] \\
1972\end{array}$ & $\begin{array}{l}\text { Retrospective } \\
1955-1969\end{array}$ & $\begin{array}{l}110 \text { recurrent or metastatic } \\
\text { CRC } \mathbf{R S N}=79 \text { treatment for } \\
\text { pelvic recurrence }\end{array}$ & 400-1750 NSDE & $\begin{array}{l}\text { Symptom relief, } \\
\text { 4-point scale }\end{array}$ & NR \\
\hline
\end{tabular}


Table I. (Continued).

\begin{tabular}{|c|c|c|c|c|c|}
\hline Author/year & $\begin{array}{l}\text { Study design and } \\
\text { treatment period }\end{array}$ & Participants & $\begin{array}{l}\text { Radiotherapy (Dose range/ } \\
\text { fraction size/treatment } \\
\text { period) }\end{array}$ & $\begin{array}{l}\text { Relevant } \\
\text { outcome }\end{array}$ & Follow-up \\
\hline $\begin{array}{l}\text { Stearns [33] } \\
1970\end{array}$ & $\begin{array}{l}\text { Retrospective } \\
1965-1968\end{array}$ & $\begin{array}{l}\mathbf{N}=\mathbf{6 1} \text { inoperable or recurrent } \\
\text { pelvic CRC }\end{array}$ & $\begin{array}{l}20-25 \mathrm{~Gy} / 2-3 \mathrm{wk} \\
\text { Several repeated courses }\end{array}$ & $\begin{array}{l}\text { Pain relief, } \\
\text { 4-point scale }\end{array}$ & $\begin{array}{l}\text { Through } \\
\text { January } \\
1968 \text { or until } \\
\text { death }\end{array}$ \\
\hline $\begin{array}{l}\text { Trotter [13] } \\
1996\end{array}$ & $\begin{array}{l}\text { RCT } \\
1985-1991\end{array}$ & $\begin{array}{l}73 \text { inoperable or recurrent rectal } \\
\text { cancer } \\
\text { RSN }=\mathbf{3 7} \text { randomized to EBRT } \\
\text { alone (control gp) }\end{array}$ & $\begin{array}{l}\text { Median } 45 \text { Gy }(16.2-54 \\
\text { Gy } / 1.5-1.8 \mathrm{~Gy} / \\
5.5 \mathrm{wk}(1.5-9)\end{array}$ & $\begin{array}{l}\text { Reduction in } \\
\text { pain score, } \\
4-5 \text {-point } \\
\text { scale }\end{array}$ & $\begin{array}{l}\text { Until } \\
\text { progression } \\
\text { or death }\end{array}$ \\
\hline $\begin{array}{l}\text { Urdaneta-Lafee } \\
\text { [34] } \\
1972\end{array}$ & $\begin{array}{l}\text { Retrospective } \\
\text { NR }\end{array}$ & $\begin{array}{l}135 \text { inoperable or recurrent } \\
\text { rectal cancer } \\
\mathbf{R S N}=\mathbf{1 0 2} \text { given EBRT alone }\end{array}$ & $10-60$ Gy/2 Gy & $\begin{array}{r}\text { Symptom relief, } \\
\text { dichotomized }\end{array}$ & $\begin{array}{l}\text { NR. } 2 \text { lost to } \\
\text { follow-up }\end{array}$ \\
\hline $\begin{array}{l}\text { Wang [35] } \\
1962\end{array}$ & $\begin{array}{l}\text { Retrospective } \\
1940-1960\end{array}$ & $\begin{array}{l}111 \text { inoperable, residual or } \\
\text { recurrent rectal, RS or } \\
\text { sigmoid cancer } \mathbf{R S N}=\mathbf{8 2} \\
\text { adequate follow-up }\end{array}$ & $<20$ Gy to $>50$ Gy & $\begin{array}{r}\text { Symptom relief, } \\
\text { dichotomized }\end{array}$ & NR \\
\hline $\begin{array}{l}\text { Williams [36] } \\
1949\end{array}$ & $\begin{array}{l}\text { Retrospective } \\
?-1946\end{array}$ & $\begin{array}{l}192 \text { rectal cancer } \\
\mathbf{R S N}=\mathbf{1 2 8} \text { inoperable (primary } \\
\text { and recurrent) }\end{array}$ & $\begin{array}{l}\text { One patient given } 50 \\
\text { Gy/4-6 wk }\end{array}$ & $\begin{array}{l}\text { Relief of } \\
\text { symptoms, } \\
\text { not defined }\end{array}$ & $\begin{array}{l}\text { NR. } 2 \text { lost to } \\
\text { follow-up }\end{array}$ \\
\hline $\begin{array}{l}\text { Williams [17] } \\
1956\end{array}$ & $\begin{array}{l}\text { Retrospective } \\
1937-1954\end{array}$ & $\mathbf{N}=\mathbf{1 8 9}$ inoperable rectal cancer & $\begin{array}{l}\text { Aim } 60 \mathrm{~Gy} / 1.5-2 \mathrm{~Gy} / 6-8 \\
\text { wk }\end{array}$ & $\begin{array}{l}\text { Relief of } \\
\text { symptoms, } \\
\text { 3-point scale }\end{array}$ & Several yr \\
\hline $\begin{array}{l}\text { Williams [37] } \\
1957\end{array}$ & $\begin{array}{l}\text { Retrospective } \\
\text { NR }\end{array}$ & $\mathbf{N}=\mathbf{8 2}$ recurrent rectal cancer & $30-60 \mathrm{~Gy} / 3-6 \mathrm{wk}$ & $\begin{array}{l}\text { Relief of } \\
\text { symptoms, } \\
\text { dichotomized }\end{array}$ & NR \\
\hline $\begin{array}{l}\text { Wise [38] } \\
1959\end{array}$ & $\begin{array}{l}\text { Retrospective } \\
\text { NR }\end{array}$ & $\begin{array}{l}\mathbf{N}=\mathbf{2 2} \text { recurrent or residual } \\
\text { rectal, } \mathrm{RS} \text { and sigmoid cancer }\end{array}$ & $\begin{array}{l}\text { Mean } 46 \text { Gy }(30-60) \\
6 \text { patients previously treated } \\
\text { with EBRT }\end{array}$ & $\begin{array}{l}\text { Pain relief, } \\
\text { 3-point scale }\end{array}$ & NR \\
\hline
\end{tabular}

CRC, colorectal cancer; EBRT, external beam radiotherapy; gp, group; Gy, Gray; MeV, mega electron volt; mo, months; NR, not reported; NSDE, nominal standard dose equivalents; PI, palliative index (symptom-free period relative to survival duration); Pro, prospective; RCT, randomized controlled trial; RS, rectosigmoid; RSN, number of patients in the relevant subgroup; wk, weeks; yr, years.

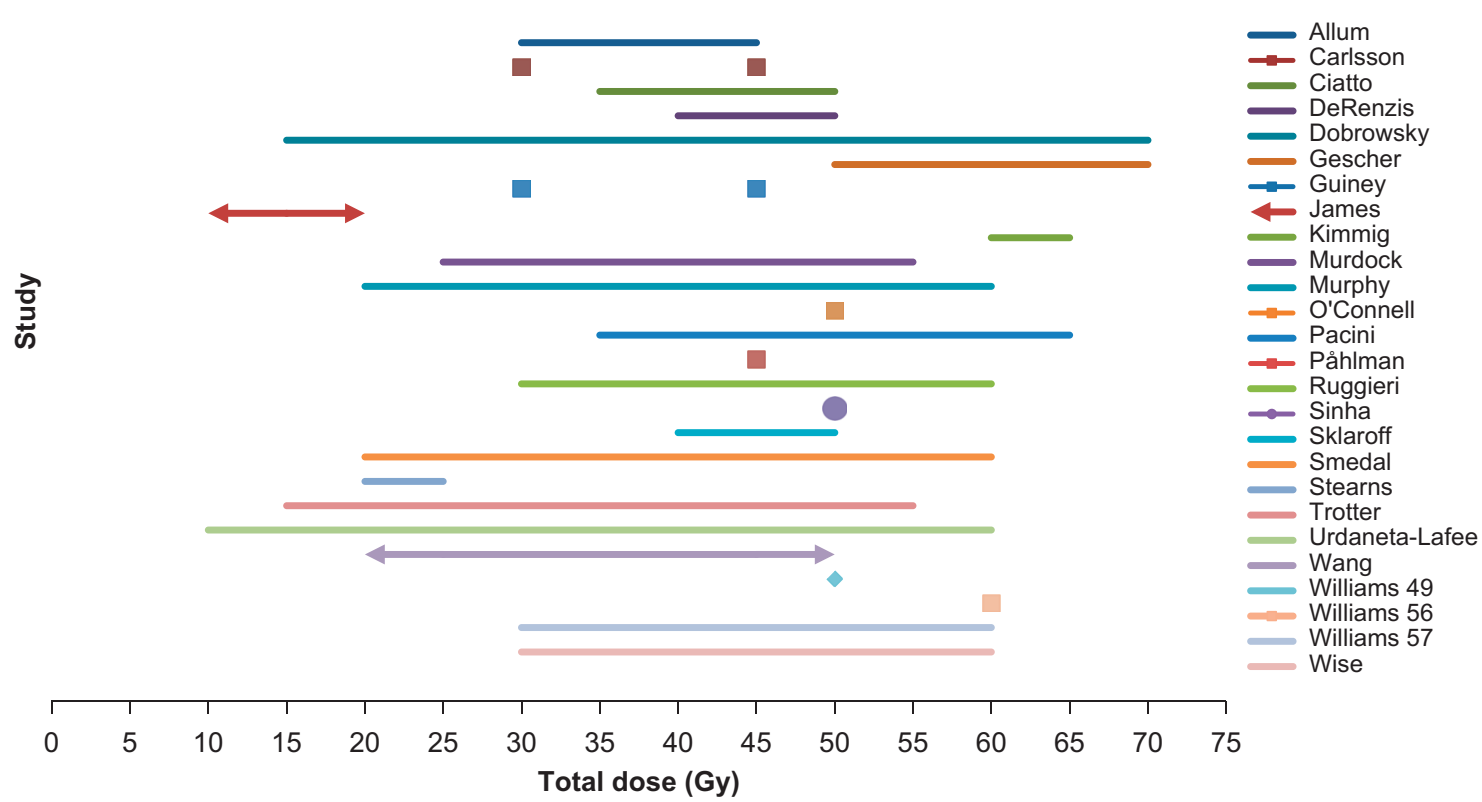

Figure 2. Arrows indicate no upper or lower limit. $\mathbf{0}=$ mean dose only. $\bullet=$ dose reported for only one patient. Study by Soleimani is not listed (dose in NSDE). 
Table II. Symptomatic response to palliative pelvic radiotherapy of rectal cancer.

\begin{tabular}{|c|c|c|c|c|}
\hline Author & $\begin{array}{l}\text { Radiation } \\
\text { dose }(G y)\end{array}$ & $\begin{array}{l}\text { Symptoms included } \\
\text { in the reported response* }\end{array}$ & $\begin{array}{c}\text { Response }^{\wedge} \\
\text { (positive responses/total) }\end{array}$ & $\begin{array}{l}\text { Duration in } \\
\text { months (range) }\end{array}$ \\
\hline Allum [19] & $5-45$ & Pain & Pain 13/19 & Median $3(1-7)$ \\
\hline Carlsson [14] & 30 or 45 & Pain & Pain 40/43 & Mean $6(2-10)$ \\
\hline Ciatto [20] & $35-65$ & $\begin{array}{l}\text { Pain, vaginal bld/dc, rectal do, } \\
\text { dysuria }\end{array}$ & $50 / 90$ & $6: 33 \%, 12: 15 \%$ \\
\hline DeRenzis [36] & $<40$ to $>50$ & $\begin{array}{l}\text { Pain, rectal bld/dc, urinary do, } \\
\text { vaginal bld/dc }\end{array}$ & $25 / 35$ & 6 (or death): $71 \%$ \\
\hline Dobrowsky [21] & $15-70$ & Pain & Pain $34 / 38$ & $\mathrm{NR}$ \\
\hline Gescher [22] & $50-70$ & $\begin{array}{l}\text { Pain, defecation do, rectal bld/dc, } \\
\text { vaginal bld, mass }\end{array}$ & 44/51; Pain 30/33 & Mean 9 (0-53), 6: 50\% \\
\hline Guiney [23] & $30-45$ & NS & $12 / 17$ & NR \\
\hline James [24] & $<10$ to $>20$ & $\begin{array}{l}\text { Pain, mass, bld/dc, urinary, } \\
\text { neurologic }\end{array}$ & $83 / 119$ & NR \\
\hline Kimmig [25] & $60-66$ & Pain & Pain 53/74 & $6-12$ \\
\hline Murdock [26] & $24-56$ & Pain, mass/ulceration, dc, edema & $5 / 9$ & R 5-18, 6: 44\%, 12: 33\% \\
\hline Murphy [37] & $20-60$ & Pain, mass & $73 / 127$ & $6: 58 \%, 12: 42 \%, 24: 8 \%$ \\
\hline O'Connell [12] & 50 & Pain & Pain $17 / 18$ & Median $5(1-44+)$ \\
\hline Pacini [27] & $35-65$ & $\begin{array}{l}\text { Pain, vaginal bld/dc, dysuria, rectal } \\
\text { do }\end{array}$ & $115 / 143$ & $6: 32 \%, 12: 13 \%, 24: 6 \%$ \\
\hline Påhlman [15] & 46 & $\begin{array}{l}\text { Bld, pain, altered bowel habit, } \\
\text { soiling, incontinence }\end{array}$ & $\begin{array}{l}\text { 26/27; Bld } 15 / 15 \text {; pain } 12 / 13 ; \\
\quad \text { altered bowel habit } 11 / 12 ; \\
\text { soiling } 9 / 10 \text {; incontinence } 1 / 3\end{array}$ & Median $5(1-20)$ \\
\hline Ruggieri [28] & $30-60$ & Pain, nodules, mass, bld & Pain $45 / 67$ & $3: 32 \%$ \\
\hline Sinha [29] & Avg 50 & $\begin{array}{l}\text { Pain, rectal bld/dc, perineal nodules, } \\
\text { vaginal dc, diarrhea, dysuria }\end{array}$ & $\begin{array}{l}\text { Pain } 13 / 23 \text {; bld } 2 / 3 \text {; dc } 4 / 6 \text {; } \\
\text { diarrhea } 2 / 2 \text {; nodules } \\
2 / 3 \text {; dysuria } 1 / 2\end{array}$ & NR \\
\hline Sklaroff [18] & $40-50$ & Bld & 10/10; Bld 10/10 & 6: $90 \%$ \\
\hline Smedal [30] & $20-60$ & Pain, bld/dc, mass & $36 / 50$ & $6:>50 \%, 12:>26 \%$ \\
\hline Soleimani [16] & $400-1750$ rets $^{\#}$ & "Pelvic syndrome"" & "Pelvic syndrome" 57/79 & NR \\
\hline Stearns [35] & $20-25$ & NS & $57 / 61$ & $6: 80 \%$ \\
\hline Trotter [13] & $16-54$ & Pain & Pain 21/37 & NR \\
\hline $\begin{array}{l}\text { Urdaneta-Lafee } \\
\text { [38] }\end{array}$ & $10-60$ & $\begin{array}{l}\text { Bld, pain, diarrhea, constipation, } \\
\text { tenesmus, urinary }\end{array}$ & $85 / 102$ & NR \\
\hline Wang[33] & $<20$ to $>50$ & Pain, mass, bld/dc & $\begin{array}{l}\text { 69/82; Pain } 63 / 76 \text {; mass } 12 / 20 \text {; } \\
\text { bld/dc } 19 / 20\end{array}$ & NR \\
\hline Williams [34] & NR & Pain, tenesmus, bld, dc, ulceration & $60 \%$ & NR \\
\hline Williams [17] & 60 & Bld, rectal dc, pain, tenesmus & $\begin{array}{l}\text { Bld } 121 / 135 \text {; dc } 77 / 116 \text {; pain } \\
\quad 78 / 102 \text {; tenesmus } 48 / 66\end{array}$ & NR \\
\hline Williams [31] & $30-60$ & $\begin{array}{l}\text { Pain, mass, dc, bld, urinary, edema, } \\
\text { diarrhea }\end{array}$ & $71 / 82$ & $3: 55 \%, 6: 32 \%, 12: 12 \%$ \\
\hline Wise [32] & $30-60$ & $\begin{array}{l}\text { Pain, mass, vaginal bld, perineal } \\
\text { abscess }\end{array}$ & $\begin{array}{l}\text { Pain } 18 / 18 \text {; mass } 5 / 5 \text {; vaginal } \\
\text { bld } 3 / 3\end{array}$ & $\begin{array}{l}\text { Mean } 4.5 \\
\quad(3 \text { weeks-18 months) }\end{array}$ \\
\hline
\end{tabular}

*Symptoms are listed in order of their reported frequencies.

^Overall symptomatic response (in bold), unless otherwise stated.

$a$, bleeding, tenesmus, discharge, pain, urinary symptoms and edema.

\#, nominal standard dose equivalents (NSDE); Bld, bleeding; dc, discharge; do, disorder; NR, not reported; NS, not specified other than as "symptoms"; R, range.

was reported in 17 articles and ranged from $56 \%$ to $100 \%$. Three studies reported $100 \%$ responses for bleeding [18,21,35].

Response criteria varied across studies, but the majority of authors defined response as symptomatic relief on a $3-5$ point scale. Two studies classified responders as having "complete regression of symptoms" $[23,32]$ while two others specified best symptomatic response $[16,26]$. With the exception of one study [17], it remained unclear whether results reflected the best response observed during the follow-up period or the response measured at a certain time point. One study used a grading scale classifying response according to duration of palliation of symptoms rather than the degree to which the symptoms were relieved [33]. Older studies tended to report narrative descriptions rather than quantitative results of treatment $[36,37]$. Four studies used the discontinuation of analgesia as a marker of treatment response [22,27,30,38]. No studies reported patient-reported outcomes (PRO) of symptom relief or description of QOL changes. 
Table III. Pooled symptomatic response rates according to authors' own definitions, at variable time points after palliative pelvic radiotherapy.

\begin{tabular}{lr}
\hline Symptom & \multicolumn{1}{c}{ Response* $^{*}$} \\
\hline Overall response, including "pelvic syndrome” & $818 / 1084=75 \%$ \\
{$[15,16,18,20,22-24,26,27,30,31,33$,} & \\
35-38] (specific symptom not indicated) & \\
Pain [12-15,17,19,21,22,25,28,29,32,33] & $437 / 561=78 \%$ \\
Bleeding and discharge $[15,17,18,29,32,33]$ & $251 / 308=81 \%$ \\
Mass and tenesmus [17,32,33] & $65 / 91=71 \%$ \\
Other (diarrhea, nodules, dysuria, etc) & $26 / 32=72 \%$ \\
{$[15,29]$} & \\
\hline
\end{tabular}

*Symptomatic responses are dichotomized as "response" or "no response".

aWilliams' 1949 study is not included in the table because the number of responses cannot be determined.

\section{Dose response}

Symptomatic responses were reported at low total doses of radiotherapy ( $\leq 20$ Gy) [36,38], during the course of fractionated treatment [16] and after single fractions of 5-10 Gy [22]. Påhlman et al. reported that palliation was observed at 20-30 Gy and that those patients without symptomatic effect at $46 \mathrm{~Gy}$ did not benefit from escalation to $64 \mathrm{~Gy}$ [18]. Several authors reported no difference in palliative effect across a range of radiotherapy prescriptions [23,24,27,30,36]. However, Soleimani et al. concluded that nominal single dose equivalents (NSDE) between 1000 and 1300 rets was the optimal range [19]. Three retrospective studies reported that the proportion of patients with longer response durations was greater among those who had received higher doses $[25,35,36]$, although they could not document a statistically significant doseresponse relationship. In contrast, James et al. found the same median duration of response both for those patients given $<15$ Gy and those given $\geq 15$ Gy. Retreatment after low dose radiotherapy was seen to be effective, particularly among those with good initial responses [38].

\section{Durability of response}

The three prospective studies reported duration of symptomatic improvement ranging from one month to more than 44 months across a range of doses from 30 to 50 Gy $[15,17,18]$. Over half of the retrospective studies reported responses lasting well over one year. Stearns et al. observed that better responses tended to last longer than poorer ones [38].

\section{Toxicity}

The toxicity of radiotherapy was addressed in 21 of 27 publications. Two of these studies evaluated toxicity prospectively, in a systematic manner $[15,16]$ and one made use of a recognized tool (WHO criteria) [16]. Toxicity was, for the most part, characterized as mild to moderate. Worst degree of toxicity reported by each of the studies is summarized in Table IV. The timing of the reported toxicities and their classification as an acute versus late was often unclear. In addition, frequencies of many of the toxicities were impossible to ascertain as several authors used descriptions such as "rare" and "some patients" rather than numerical results.

\section{Discussion}

All 27 studies included in this review reported that radiotherapy was effective in palliating pelvic symptoms such as pain, bleeding, and mass effect, without reports of unacceptable toxicity. However, considerable heterogeneity in patients, treatment, and outcomes reported, and methodological shortcomings among the majority of studies, limits the reliability and generalizability of their results. This systematic review demonstrates the paucity of valid documentation and the need for prospective trials analyzing the benefit and harm of modern palliative radiotherapy among patients with symptomatic rectal cancer.

The vast majority of included studies were retrospective chart reviews with inherent methodological deficiencies. Follow-up was often variable and data incomplete, in populations that reflected disparities in clinical practices. Definitions of key concepts such as "palliative intent" and "advanced disease" as well as definitions of what is deemed medically inoperable or surgically unresectable were not standardized across the included studies and have evolved over time. Duration of response could not be determined in many of the studies due to retrospective review of non-systematic clinical follow-up. None of the included studies adequately described treatments such as analgesics and chemotherapy, which may have confounded their results. In addition, data collection in some studies spanned several decades [23] during which time there was considerable variability in the method of radiotherapy delivery and potential co-interventions. Hence, we cannot reach firm conclusions regarding the effect of palliative pelvic EBRT, and the validity of published results can also be questioned. Risk of publication bias, inherent to the review process itself, should be taken into account when interpreting the results of this review.

Conducting research on palliative radiotherapy of rectal cancer is difficult, as reflected in the reviewed studies. Challenges include limiting the potential confounding interventions, applying uniform interventions across a population of incurable patients, 
Table IV. Toxicity reported in studies of palliative pelvic radiotherapy of rectal cancer.

\begin{tabular}{|c|c|c|c|}
\hline Author & Gastrointestinal & Genitourinary & Skin/connective tissue \\
\hline Allum [19] & mild* & NR & NR \\
\hline Carlsson [14] & none observed & none observed & none observed \\
\hline Ciatto $[20]$ & mild, no late & NR & mild \\
\hline DeRenzis [36] & mild & mild & mild \\
\hline Dobrowsky [21] & mild & mild & mild \\
\hline Gescher [22] & enteritis requiring operation $2 / 51$ & NR & mild $23 / 51$ \\
\hline Guiney [23] & moderate $^{* *} 3 / 27$, no late & NR & moderate $1 / 27$ \\
\hline James [24] & mild $13 / 119$, severe ${ }^{* * *} 8 / 119$ & NR & NR \\
\hline Murdock [26] & NR & NR & mild \\
\hline O'Connell [12] & $\begin{array}{l}\text { mild - moderate } \\
\text { SBO } 3 / 19\end{array}$ & NR & NR \\
\hline Pacini [27] & mild & NR & mild \\
\hline Sinha [29] & $\begin{array}{l}\text { mild - moderate } 9 / 25 \\
\text { adhesions } 1 / 25, \text { SBO } 1 / 25\end{array}$ & NR & $\begin{array}{l}\text { mild } 1 / 25 \\
\text { fibrosis } 1 / 25\end{array}$ \\
\hline Sklaroff [18] & mild & NR & fibrosis $3 / 10$ \\
\hline Smedal [30] & severe $4 / 50$ & anuria (pelvic scarring) $1 / 50$ & NR \\
\hline Stearns [35] & mild & NR & $\mathrm{NR}$ \\
\hline Trotter [13] & $11 / 37$ grade 3 or 4 (WHO criteria) & NR & NR \\
\hline Urdaneta-Lafee [37] & mild - moderate & NR & NR \\
\hline Williams [34] & $\begin{array}{l}\text { mild - moderate } \\
\text { bowel reactions } 24 / 192 \text {, pain } 6 / 192, \\
\text { fistula } 12 / 192 \text {, stricture } 32 / 192\end{array}$ & moderate $19 / 192$ & $\begin{array}{l}\text { mild } \\
\text { necrosis } 6 / 192\end{array}$ \\
\hline Williams [17] & $\begin{array}{l}\text { mild - moderate } \\
\text { rectal stenosis } 1 / 189 \text {, fistula } 12 / 189\end{array}$ & mild - moderate & $\begin{array}{l}\text { mild - moderate } \\
\text { fibrosis } 32 / 189 \text {, necrosis } 9 / 189\end{array}$ \\
\hline Williams [31] & mild - moderate & moderate & $\begin{array}{l}\text { severe } \\
\text { necrosis } 2 / 82\end{array}$ \\
\hline Wise [32] & mild - moderate $11 / 22$, severe $3 / 22$ & moderate $2 / 22$ & moderate - severe $5 / 22$ \\
\hline
\end{tabular}

Late complications are indicated in bold type text.

NR, not reported; SBO, small bowel obstruction; WHO, World Health Organization.

*Mild indicates that the author has described the toxicity as "mild" or as not requiring more than symptomatic measures.

** Moderate indicates that the author has described the toxicity as "moderate" or that the treatment was interrupted due to toxicity.

***Severe indicates that the author has described the toxicity as "severe" or that the treatment was discontinued due to toxicity.

and obtaining repeated, valid outcome measures, with sufficient length of follow-up, from patients with progressive cancer.

However, despite the inherent limitations, the pooled overall response rate of $75 \%$ among 1084 cases seems to be of clinical importance. In addition, specific target symptoms including pain, bleeding, discharge and mass effect all responded to palliative pelvic radiotherapy, with pooled results ranging from $71 \%$ to $81 \%$. Surprisingly, we found no studies fitting our inclusion criteria that assessed palliative pelvic radiotherapy of rectal cancer within the last 20 years. During this time, significant advances have been made in diagnostic imaging, radiotherapy planning, and multimodal treatment of rectal cancer. However, despite the relatively crude methodology of these historical series, there appears to be a consistently positive treatment effect. More modern studies of preoperative radiotherapy have demonstrated radiologic down-staging among a similar proportion of patients $(74 \%-82 \%)$ treated with relatively low total doses $(5 \mathrm{~Gy} \times 5)[8,9]$. There are also reports of symptomatic improvement in such curative treatment settings [7].
In the reviewed studies, the severity, cumulative incidence and duration of toxicities reported cannot be accurately interpreted and are most likely underestimated. Among patients with advanced rectal cancer, it may also be difficult to differentiate side effects of radiotherapy from symptoms of progressive disease $[15,37,39]$. While developments in radiotherapy planning and delivery have reduced the risks of acute side effects [40], the risk of late complications remains uncertain in today's patients considering that modern systemic palliative treatment of metastatic colorectal cancer has increased the duration of survival of this group of patients [1].

In non-randomized studies, such as the majority of those included in this review, the prescribed radiotherapy dose and fractionation regimen is likely to stratify patients according to functional status. Healthier patients with presumed better tolerance were likely given larger target doses than sicker patients [21,22,25-27,30,35,36,41]. Although several studies claimed to demonstrate that duration of symptomatic response increased with dose, significant risk of selection bias 
precluded valid conclusions regarding target doses, optimal fractionation schemes and dose-response relationships.

The burden of time spent in treatment and the risk of increased side effects with higher radiotherapy doses should not be imposed on patients in a palliative situation without sufficient evidence of its benefit. Single fractions or hypofractionated prescriptions could be particularly meaningful for these patients with limited life expectancies, by reducing the burden of treatment.

Patients with rectal cancer beyond cure at presentation or recurrence, patients who are medically unfit for surgery or multimodal oncological treatment, and patients who for other reasons choose not to undergo radical treatment, continue to be in need of effective symptom palliation. Palliative pelvic radiotherapy appears to provide an important contribution to the armamentarium of palliative treatments for these patients. Unfortunately, there is a lack of prospective studies performed with modern radiotherapy planning systems and relevant endpoints in this area and consequently, we have only weak evidence on which to base our treatment decisions. Hence, we are currently conducting a prospective study of palliative EBRT of rectal cancer in eight of nine radiotherapy centers across Norway (ClinicalTrials.gov Identifier NCT01023529).

\section{Conclusion}

The reviewed studies consistently report effective palliation of symptomatic incurable rectal cancer across a range of radiotherapy schedules. However, due to methodological shortcomings in the reports and great inter-study variability, it is impossible to draw valid and reliable conclusions regarding the onset, duration or degree of the palliative effect, or potential toxicity. Prospective studies, using modern radiotherapy planning systems and standardized palliative dose radiotherapy regimens, systematically addressing relevant outcomes are needed to clarify the effect of palliative radiotherapy of rectal cancer [42]. Studies should include patient-defined target symptoms, validated research tools and consider major confounding factors such as concomitant anticancer and palliative interventions.

Declaration of interest: The authors report no conflicts of interest. The authors alone are responsible for the content and writing of the paper.

This project was supported by a research grant from the South-Eastern Norway Regional Health Authority.

\section{References}

[1] Glimelius B, Cavalli-Bjorkman N. Metastatic colorectal cancer: Current treatment and future options for improved survival. Medical approach - present status. Scand J Gastroenterol 2012;47:296-314.

[2] Poultsides GA, Servais EL, Saltz LB, Patil S, Kemeny NE, Guillem JG, et al. Outcome of primary tumor in patients with synchronous stage IV colorectal cancer receiving combination chemotherapy without surgery as initial treatment. J Clin Oncol 2009;27:3379-84.

[3] Jacob S, Wong K, Delaney GP, Adams P, Barton MB. Estimation of an optimal utilisation rate for palliative radiotherapy in newly diagnosed cancer patients. Clin Oncol 2010;22:56-64.

[4] Delaney G, Jacob S, Featherstone C, Barton M. The role of radiotherapy in cancer treatment: Estimating optimal utilization from a review of evidence-based clinical guidelines. Cancer 2005;104:1129-37.

[5] Chow E, Zeng L, Salvo N, Dennis K, Tsao M, Lutz S. Update on the systematic review of palliative radiotherapy trials for bone metastases. Clin Oncol 2012;24:112-24.

[6] Lutz ST, Chow EL, Hartsell WF, Konski AA. A review of hypofractionated palliative radiotherapy. Cancer 2007;109:1462-70.

[7] Radu C, Berglund A, Pahlman L, Glimelius B. Short-course preoperative radiotherapy with delayed surgery in rectal cancer - a retrospective study. Radiother Oncol 2008; 87:343-9.

[8] Pettersson D, Holm T, Iversen H, Blomqvist L, Glimelius B, Martling A. Preoperative short-course radiotherapy with delayed surgery in primary rectal cancer. Br J Surg 2012; 99:577-83.

[9] Hatfield P, Hingorani M, Radhakrishna G, Cooper R, Melcher A, Crellin A, et al. Short-course radiotherapy, with elective delay prior to surgery, in patients with unresectable rectal cancer who have poor performance status or significant co-morbidity. Radiother Oncol 2009;92:210-4.

[10] Wong R, Thomas G, Cummings B, Froud P, Shelley W, Withers $\mathrm{RH}$, et al. The role of radiotherapy in the management of pelvic recurrence of rectal cancer. Can J Oncol 1996; 6(Suppl 1):39-47.

[11] Benson AB, 3rd, Guillem JG, Minsky BD. Have the changes in treatment of rectal cancer made a significant difference to our patients? Oncology 2011;25:1323-9.

[12] Liberati A, Altman DG, Tetzlaff J, Mulrow C, Gotzsche PC, Ioannidis JP, et al. The PRISMA statement for reporting systematic reviews and meta-analyses of studies that evaluate health care interventions: Explanation and elaboration. J Clin Epidemiol 2009;62:e1-34.

[13] Higgins JPT, Green S, editors. Cochrane handbook for systematic reviews of interventions. Version 5.0.2. The Cochrane Collaboration 2009.

[14] Sanderson S, Tatt ID, Higgins JP. Tools for assessing quality and susceptibility to bias in observational studies in epidemiology: A systematic review and annotated bibliography. Int J Epidemiol 2007;36:666-76.

[15] O'Connell MJ, Childs DS, Moertel CG, Holbrook MA, Schutt AJ, Rubin J, et al. A prospective controlled evaluation of combined pelvic radiotherapy and methanol extraction residue of BCG (MER) for locally unresectable or recurrent rectal carcinoma. Int J Radiat Oncol Biol Phys 1982; 8:1115-9.

[16] Trotter JM, Edis AJ, Blackwell JB, Lamb MH, Bayliss EJ, Shepherd JM, et al. Adjuvant VHF therapy in locally recurrent and primary unresectable rectal cancer. Aust Radiol 1996;40:298-305. 
[17] Carlsson G, Hafstrom L, Jonsson PE, Ask A, Kallum B, Lunderquist A. Unresectable and locally recurrent rectal cancer treated with radiotherapy or bilateral internal iliac artery infusion of 5-fluorouracil. Cancer 1986;58:336-40.

[18] Pahlman L, Glimelius B, Ginman C, Graffman S, Adalsteinsson B. Preoperative irradiation of primarily nonresectable adenocarcinoma of the rectum and rectosigmoid. Acta Radiol Oncol 1985;24:35-9.

[19] Soleimani PK, Hindo WA, Hendrickson FR. Dose-response relationship in radiation therapy of advanced carcinoma of the colon and rectum. Radiology 1972;103:179-81.

[20] Williams IG, Horwitz H. The primary treatment of adenocarcinoma of the rectum by high voltage roentgen rays $(1,000 \mathrm{kv})$. Am J Roentgenol Radium Ther Nucl Med 1956;76:919-28.

[21] Sklaroff DM. Radiation as primary therapy for rectal carcinoma. Am Fam Physician 1973;8:81-5.

[22] Allum WH, Mack P, Priestman TJ, Fielding JW. Radiotherapy for pain relief in locally recurrent colorectal cancer. Ann R Coll Surg Engl 1987;69:220-1.

[23] Ciatto S, Pacini P. Radiation therapy of recurrences of carcinoma of the rectum and sigmoid after surgery. Acta Radiol Oncol 1982;21:105-9.

[24] Dobrowsky W, Schmid AP. Radiotherapy of presacral recurrence following radical surgery for rectal carcinoma. Dis Colon Rectum 1985;28:917-9.

[25] Gescher FM, Keijser AH. [Palliative radiotherapy of recurrence in rectal carcinoma]. Ned Tijdschr Genees 1987;131:533-5.

[26] Guiney MJ, Smith JG, Worotniuk V, Ngan S. Results of external beam radiotherapy alone for incompletely resected carcinoma of rectosigmoid or rectum: Peter MacCallum Cancer Institute experience 1981-1990. Int J Radiat Oncol Biol Phys 1999;43:531-6.

[27] James RD, Johnson RJ, Eddleston B, Zheng GL, Jones JM. Prognostic factors in locally recurrent rectal carcinoma treated by radiotherapy. Br J Surg 1983;70:469-72.

[28] Kimmig B, Engenhart R, Flentje M, Marin-Grez M, Hover KH. [Radiotherapy using photons and neutrons in recurrences of colorectal tumors]. Rontgen-Blatter 1989; 42:47-50.

[29] Murdock MG, Kramer S. Cobalt 60 therapy in the management of perineal recurrence from carcinoma of rectum and colon. Am J Roentgenol Radium Ther Nucl Med 1964;91:149-54.
[30] Pacini P, Cionini L, Pirtoli L, Ciatto S, Tucci E, Sebaste L. Symptomatic recurrences of carcinoma of the rectum and sigmoid. The influence of radiotherapy on the quality of life. Dis Colon Rectum 1986;29:865-8.

[31] Grillo Ruggieri F, Siragusa A. [Radiotherapy of recurrences of adenocarcinoma of the rectum after radical surgery: Galliera Hospitals (1976-1985)]. La Radiol Med 1989; 78:363-6.

[32] Sinha PP. Radiation therapy with/without simultaneous weekly 5 F.U. in locally recurrent carcinoma of the recto-sigmoid. W V Med J 1989;85:137-41.

[33] Smedal MI, Wright KA, Siber FJ. The palliative treatment of recurrent carcinoma of rectum and rectosigmoid with $2 \mathrm{mv}$. radiation. Some results and description of a technique. Am J Roentgenol Radium Ther Nucl Med 1967;100:904-8.

[34] Williams IG, Shulman IM, Todd IP. The treatment of recurrent carcinoma of the rectum by supervoltage $\mathrm{x}$-ray therapy. Br J Surg 1957;44:506-8.

[35] Wise RE, Smedal MI. Palliative treatment of recurrent rectosigmoidal neoplasms with two million volt radiation. Surg Clin North Am 1959;39:775-80.

[36] Wang CC, Schulz MD. The role of radiation therapy in the management of carcinoma of the sigmoid, rectosigmoid, and rectum. Radiology 1962;79:1-5.

[37] Williams IG. Carcinoma of the rectum and anal canal; treatment with very high voltage x-ray therapy. Br J Surg 1949;36:376-81.

[38] Stearns MW, Jr., Whiteley HW, Jr., Leaming RH, Deddish MR. Palliative radiation therapy in patients with localized cancer of the colon and rectum. Dis Colon Rectum 1970; 13:112-5.

[39] Urdaneta-Lafee N, Kligerman MM, Knowlton AH. Evaluation of palliative irradiation in rectal carcinoma. Radiology 1972;104:673-7.

[40] Bernier J, Hall EJ, Giaccia A. Radiation oncology: A century of achievements. Nat Rev Cancer 2004;4:737-47.

[41] De Renzis C, Gaeta M, Frosina P, Bucolo A, Raffaele L. Techniques and results in the irradiation of recurrences, macroscopic residues, and primary inoperable tumors in rectal cancer. Rays 1986;11:119-23.

[42] Cameron MG, Kersten C, van Helvoirt R, Rohde G, Fossa SD, Vistad I. Patient reported outcomes of symptoms and quality of life among cancer patients treated with palliative pelvic radiation: A pilot study. BMC Res Notes 2011;4:252. 